\title{
Atomic Force Microscopy Investigation of Influenza A Virus Nuclear Export Protein Aggregation
}

\author{
EV Dubrovin ${ }^{1,2^{*}}$, ON Koroleva $^{1}$, AO Golovko ${ }^{1}$, NV Kuzmina $^{3}$, DV Klinov $^{2}$ and VL Drutsa ${ }^{1}$ \\ 1. Lomonosov Moscow State University, Leninskie gory, 1-2, Moscow, Russian Federation. \\ 2. Federal Research and Clinical Center of Physical-Chemical Medicine of Federal Medical Biological \\ Agency, Malaya Pirogovskaya, 1a, Moscow, Russian Federation. \\ 3. Frumkin Institute of Physical Chemistry and Electrochemistry, Russian Academy of Sciences, \\ Moscow, Russia. \\ * Corresponding author: dubrovin@polly.phys.msu.ru
}

The $14.5 \mathrm{kDa}$ nuclear export protein (NEP) is essential for the influenza A virus life cycle [1]. In particular, NEP mediates interconnection between the CRM1 nuclear export pathway and M1-viral ribonucleoprotein (vRNP) complexes, which is used for vRNP export from the host cell nucleus to the cytoplasm. Understanding of physicochemical properties of NEP may contribute to better understanding of its functioning and is, therefore, of vital importance. Recently we have shown that NEP is prone to aggregation with formation of aggregates with various dimensions ranging from tens of nanometers to microns [2]. The aim of this study was to characterize the morphology of different NEP aggregates using atomic force microscopy (AFM).

For this work NEP was expressed in E. coli cells and purified by affinity chromatography as described earlier [3]. For AFM investigation of NEP aggregates the protein solution was deposited onto a substrate surface such as modified mica and highly oriented pyrolytic graphite. AFM measurements were performed in ambient environment using the multimode atomic force microscope Nanoscope 3a (Digital instruments, USA) operated in tapping mode.

AFM images have revealed a variety of structures formed by NEP. In particular, they include globular, wormlike and rodlike aggregates of different height ranging from several nanometres to several tens nanometres (some examples of aggregates of wild type NEP are presented in Fig. 1a, and their height distribution is shown in Fig. 1b). Moreover, NEP has formed a precipitate that is composed of micronssized aggregates (Fig. 1c).

The first (biggest) maximum at the distribution of heights of the observed structures $(\sim 1.5 \mathrm{~nm}$, fig. $1 \mathrm{~b})$ may correspond to the monomeric form of the protein, whereas the higher values - to the different NEP aggregates. AFM has resolved the beaded or helical substructure of the wormlike and rodlike aggregates, which was similar to the structures formed by other proteins, e.g., E. coli RNA polymerase sigma(70) subunit [4]. The observed morphological characteristics are typical for protein amyloid structures [5]. Therefore, we assume that NEP is prone to amyloid aggregation, and different types of aggregates observed in AFM images may be indicative of the different stages or pathways of this process. In particular, large NEP conglomerates (such as in Fig. 1c) may be formed due to aggregation of smaller protein aggregates.

The morphology of the structures formed by NEP tagged with a His(6) module from either N-end (NEP$\mathrm{N}$ ) or C-end (NEP-C) of a protein molecule (these tags are often used for protein separation) has been compared with the untagged protein. It has been demonstrated that the presence of His(6) modules may 
significantly change the fraction and morphological characteristics of the particular types of NEP aggregates, especially wormlike ones.

Finally, we have demonstrated that different mutations in the NEP structure have also caused the change in the number and shape of the aggregates formed by the protein.

The obtained results suggest that NEP may experience aggregation during the influenza A virus life cycle that may be important for understanding of its functioning. Moreover, the influence of chemical modifications and mutations of NEP structure on aggregation of this protein may be of interest in biotechnological applications [6].

References:

[1] AJ Eisfeld, G Neumann and Y Kawaoka, Nat. Rev. Microbiol. 13 (2015), p. 28.

[2] AO Golovko et al., Biochem. Mosc. 83 (2018), p. 1411.

[3] AO Golovko, ON Koroleva and VL Drutsa, Biochem. Mosc. 82 (2017), p. 1529.

[4] ON Koroleva et al., Soft Matter 12 (2016), p. 1974.

[5] J Adamcik and R Mezzenga, Curr. Opin. Colloid Interface Sci. 17 (2012), p. 369.

[6] The authors acknowledge funding from the Russian Science Foundation [17-75-30064].
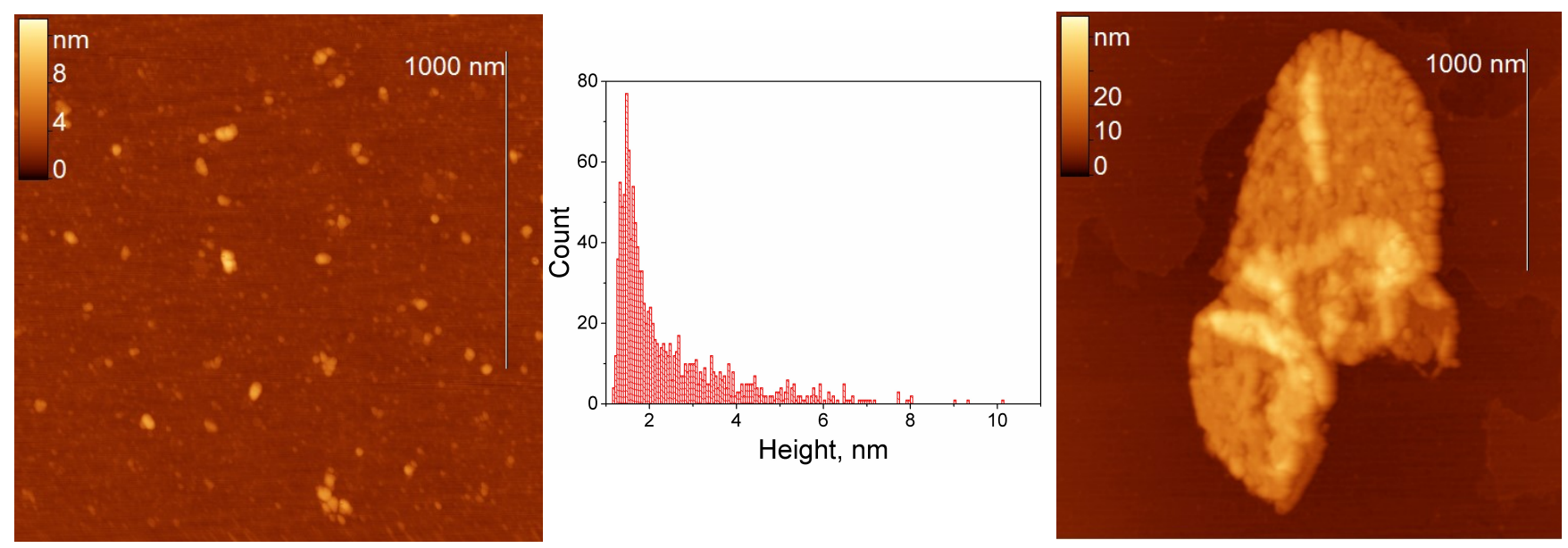

Figure 1. (a,c) Height AFM images and (b) height distribution histograms of NEP molecules and their aggregates. 